\title{
ANNOUNCEMENT 2019 Reviewer Acknowledgements
}

The Editors of Pediatric Research thank the following dedicated individuals who reviewed manuscripts in 2019.

\begin{tabular}{|c|c|}
\hline Aagaard, Kjersti & Barker, Alan R. \\
\hline Abdul-Khaliq, Hashim & Barkoski, Jacqueline \\
\hline Abel, Gregory & Barkovich, James \\
\hline Abitbol, Carolyn & Barone, Maria Vittoria \\
\hline Abman, Steve & Barrero Castillero, Alejandra \\
\hline Aceti, Arianna & Barrington, Keith \\
\hline Adibi, Jennifer & Bartock, Sarah \\
\hline Agostinis-Sobrinho, Cesar & Bartolák-Suki, Erzsébet \\
\hline Agwu, Allison & Bastian, Thomas \\
\hline Aiken, Catherine & Basu, Rajit \\
\hline Akshurin, Oleh & Bateman, Brian \\
\hline Alba, Juan Jesus Fernández & Battersby, Cheryl \\
\hline Al Alam, Denise & Batton, Beau \\
\hline Albertine, Kurt & Batu, Ezgi \\
\hline Alcorn, Joseph & Baum, Michel \\
\hline Aldrovandi, Grace M. & Beam, Kristyn \\
\hline Alfolan, Adeleye & Bearer, Cynthia \\
\hline Alkuraya, Fowzan & Beckel, Jonathan M. \\
\hline Allegaert, Karel & Bedoyan, Jirair K. \\
\hline Allegaert, Karel & Beharry, Kay \\
\hline Aloi, Marina & Behrooz, Leili \\
\hline Alsaedi, Saad & Beken, Serdar \\
\hline Alter, Blanche & Belfort, Mandy \\
\hline Aly, Hany & Bell, Katherine \\
\hline Amandito, Radhian & Bellarosa, Cristina \\
\hline Ambalavanan, Namasivayam & Bellin, Milena \\
\hline Ambinder, Rich & Bellinger, David \\
\hline Amin, Sanjiv & Below, Jennifer E. \\
\hline Anderson, Peter & Bendix, Ivo \\
\hline Anderson, Sarah & Benzies, Karen \\
\hline Andersson, Ola & Bergstrom, Nancy \\
\hline Andrade, Anderson Joel Mar- & Bernal, W. \\
\hline tino & Berni Canani, Roberto \\
\hline Andreu-Fernandez, Vicente & Bernstein, David \\
\hline Annink, Kim & Berrington, Janet \\
\hline Arboleya, Silvia & Bethell, Christina \\
\hline Ardalan, Maryam & Bhat, Ballambattu Vishnu \\
\hline Arteaga Cabeza, Olatz & Bhattacharjee, Jashdeep \\
\hline Arts, Rob J. W. & Bhattacharya, Soumyaroop \\
\hline Asghari, Golaleh & Bhatti, Faizah \\
\hline Askar, Tunay K. & Bhutani, Vinod \\
\hline Askenazi, David & Bien, Ewa \\
\hline Assa, Amit & Bilkey, Gemma \\
\hline Attia, Enas & Biniwale, M.D., Manoj \\
\hline Auvin, Stephane & Biniwale, Manoj \\
\hline Azab, Seham & Binstadt, Bryce \\
\hline Ba'Ath, Muhammad Eyad & Birken, Catherine S. \\
\hline Baccei, Mark & Bischoff, Joyce \\
\hline Bacchetta, Justine & Bizzarro, Matt \\
\hline Bailey, Helen & Black, Jane \\
\hline Balaj, Leonora & Black, Maureen M. \\
\hline Balfour, Hank & Blackwell, Caroline \\
\hline Ballard, Phillip & Blanco, Cynthia \\
\hline Balshaw, Robert & Bliss, Joseph \\
\hline Bancalari, Eduardo & Bloomfield, Frank \\
\hline Banerjee, Sanjay & Blount, Benjamin \\
\hline Bank, Ilana & Boardman, James \\
\hline
\end{tabular}

Bodelier, Alexander G. L.

Boelens, Jaap Jan

Bonifacio, Sonia

Bonkowsky, Joshua

Bonomi, Marco

Bont, Louis

Bonthius, Daniel

Booth, Garrett

Boppana, Suresh

Bora, Reeta

Borja, Sharon

Bose, Sonali

Boskabadi, Hassan

Bosse, Harold van

Boustany, Rose-Mary

Bowman, Ulrich

Boylan, Geraldine

Boyle, Kristen

Bradley, Todd

Brady, Jennifer $M$.

Brady, Kenneth

Bragg, Jennifer

Bratlid, Dag

Brecelj, Jernej

Brieño-Enriquez, Miguel Angel

Brinciotti, Mario

Brinkmeier, Heinrich

Britt, William

Brophy, Patrick

Brown, Laura

Brownell, Jefferson

Brucato, Antonio

Bruno, Claudio

Bruno, Katelyn

Buchhorn, Reiner

Buchwald, Andrea

Buhimschi, Catalin

Buhimschi, Irina A.

Bührer, Christoph

Burns, Ryan

Burrin, Douglas

Burris, Heather

Burton, Graham J.

Busch, David

Cacho, Nicole

Cadet, Jean Lud

Cakir, Ufuk

Calder, Philip

Callegari, Maria Luisa

Calzada, Raúl

Campanozzi, Angelo

Campanozzi, Angelo

Cantey, Joseph

Cardenas, Andres

Cardoso, Marly Augusto

Carlo, Waldemar

Carlson, Susan
Carpenter, Ashley

Carroll, John

Carsley, Sarah

Carter, Caitlin

Carter, Colin

Casavant, Sharon G.

Castaldi, Biagio

Castori, M.

Cavallarin, Laura

Cavero-Carbonell, Clara

Cayabyab, Rowena

Cayir, Atilla

Cedzynski, M.

Celec, Peter

Chaaban, Hala

Chafer, Consuelo

Chakkarapani, Elavazhagan

Chakraborty, M.

Chalak, Lina

Chambers, Christina

Chan, Yee-Ming

Chandrasekharan, Praveen

Chang, Todd

Chang, Yun Sil

Charlton, Jennifer

Chavez-Valdez, Raul

Chawla, Sanjay

Chemtab, Sylvain

Chemtob, Sylvain

Chen, Cheng-Nan

Chen, Yujie

Cheong, Jeanie

Chernikova, Diana

Cheung, Michael

Cheung, Wilson

Cheung, Po-Yin

Chirico, Gaetano

Chock, Valerie

Christensen, Robert

Christesen, Henrik

Christou, Helen

Chugani, Harry T.

Chung, Melissa

Cimaz, Rolando

Ciuca, Andrada

Claris, Olivier

Clark, Reese $\mathrm{H}$.

Class, Quetzal

Classen, Carl-Friedrich

Claure, Nelson

Cleal, Jane

Clyman, Ronald

Coates, Allan

Cohen, Mitchell

Colditz, Paul

Connor, Kristin

Cooney, Ashley 
Coppede, Fabio

Cornet, Marie Coralie

Correa, Adolfo

Corvaglia, Luigi

Costine, Beth

Cotten, Michael

Counsell, Serena

Couroucli, Xanthi

Cox, Bianca

Crider, Krista

Cumberland, Angrla

Cunningham, Kenda

Cunningham, S. A.

Curatolo, Paolo

Cusick, Sarah

Cutfield, Wayne

Cuttini, Marina

Czernik, Christoph

Dagle, John

D’Angelo, Gabriella

Danhaive, Olivier

Dani, Carlo

Dani, Carlo

Daniels, Stephan R

Dargaville, Peter

Darlow, Brian

Davenport, Mark

Davis, Jonathan

Dawson, Jennifer A.

de la Cruz, Diomel

de Lima, Luiz

de Meij, Tim

De Plaen, Isabelle

De Vries, Linda

Dearden, Laura

Debanne, Sara

DeFreitas, Marissa

Delgado Olguin, Paul

Dellaca', Raffaele

Delos, John

Dempsey, Eugene

Denning, Patricia Lanee Wei

Dentz, Amelie

deRegnier, Raye Ann

Derraik, Jose

Desai, Jay

Deshpande, Girish

Dharmadhikari, Avinash

Dhudasia, Miren

Di Fiore, Juliann

Diamanti, Antonella

Dianatinasab, Mostafa

Diaz, Julio

Dieterich, Douglas T.

Dijk, Peter H.

Dillstrom, Diana

Ding, Jie

Dominguez-Villar, Margarita

Donohoue, Patricia

Donowitz, Jeffrey

Doody, Daniel

Dowling, David

Drake, Amanda

Driscoll, Amanda

Dubois, Nancy

Duerden, Emma

Dumpa, Vikramaditya

Duncan, Andrea
Durand, Manuel

Dursun, Ali

Dzhala, Volodymyr

Dzietko, Mark

Eaton, Simon

Edwards, Erika

Eerden, Bram C. J. van der

Ehrmann, Stephan

Eichhorn, Joachim

Eildermann, Katja

Einspieler, Christa

Ek, Joakim

El Metwally, Dina

Elahi, Shokrollah

El-Dahr, Samir

Elder, Dawn

Eleftheriou, Despina

Elovainio, Marko

Embleton, Nicholas

Embleton, Nicholas

Emerim Lemos, Natalia

Endesfelder, Stefanie

Ericksson, Mats

Esbenshade, Adam

Esposito, Gianluca

Evans, Nicholas

Fadel, Cicely

Fadrowski, Jeffrey

Fairchild, Karen

Fajardo, Carlos

Falcone, Carmen

Falk, Bareket

Fang, Rendong

Felderhoff-Mueser, Ursula

Fenton, Tanis

Fernandes, Romulo

Fernandez-Millan, E.

Fernandez-Ruiz, Juan

Fields, David

Figeys, Daniel

Filler, Guido

Filocamo, Giovanni

Fineman, Jeffrey $R$.

Finer, Neil

Finnell, Richard

Fiorucci, Stefano

Firestein, $M$.

Firth, Amy

Fitzgerald, Maria

Flahault, Adrien

Flask, Chris

Fleishman, Rachel

Fleiss, Bobbi

Flemmer, Andreas

Fluss, Joel

Flynn, Joseph

Foley, Joan

Forster, Catherine

Fortney, Christine

Fowokan, Adeleke O.

Francavilla, Ruggiero

Frank, Deborah

Fraser, Mhoyra

Frey, Sean M.

Friedrich, Carsten

Frost, Brandy

Frymoyer, Adam

Fuentes-Afflick, Elena
Gabrielsson, S.

Gaderisi, Alfonso

Gale, Cheryl

Gale, Christopher

Galindo, Raphael

Galley, Jeffrey

Gano, Dawn

Gartstein, Maria

Gauda, Estelle B.

Gausepohl, Hans-Juergen

Gauthier, Theresa

Gavin, James P.

Gazzin, Silvia

George, Joanne

Geraghty, Patrick

Geyer, Christopher B.

Ghidini, Alessandro

Giamarellos-Bourboulis, Evan-

gelos

Gianni, Maria

Gianni, Maria L.

Giannoni, Eric

Gibson, Paul

Gien, Jason

Gierten, Jakob

Gilboa, Suzanne

Giles, Seamus

Gill, Hannah

Gill, Michelle

Gille, Christian

Gillman, Matthew

Giralt, Marta

Girchenko, Polina

Gire, Catherine

Gisselson, Marie

Gist, Katja

Gjelsvik, Annie

Gkazi, Athina

Gladstone, Melissa

Glass, Hannah

Godoy, Filipa

Goldstein, Mitchell

Gomes, Rodrigo Mello

Gonzalez-Gross, Marcela

Good, Misty

Gorenflo, Matthias

Gosain, Ankush

Gospe, Sidney

Goswami, Ipsita

Goto, Yu-ichi

Gould, Jeffrey

Govender, Yashini

Govindan, Rathinaswamy

Govindaswami, Balaji

Grammatikopoulos, Tassos

Greaves, Ronda

Greenberg, Jason

Greenough, Anne

Greer, Frank

Gregori, Silvia

Gregory, Kimberly

Greisen, Gorm

Greisen, Gorm

Gressens, Pierre

Gridneva, Zoya

Groenendaal, Floris

Gronbaek, Henning

Groves, Alan
Grunau, Ruth

Grunebaum, Eyal

Guan, Wei-Jie

Guarnizo-Herreno, Carol C.

Guedes, Dartagnan Pinto

Gueimonde, Miguel

Guerra, Vitor

Gulhan, Bora

Gundogdu, Zuhal

Gunn, Alistair

Gupta, Munish

Gurka, Matthew J.

Guzzetta, Andrea

Guzzo, Isabella

Hackam, David

Hade, Erinn

Hadzic, Dino

Haiden, Nadja

Hair, Amy

Hales, Derek

Hall, Judith G.

Hallman, Mikko

Hamada, Hiromichi

Hambleton, Sophie

Hamvas, Aaron

Han, Ke

Hanna, Mina

Hannes, Tobias

Hansen, Terkel

Hansen, Thor W. R.

Hantos, Zoltan

Hanudel, Mark

Harari, Gabriella

Harding, C. Harding

Harigopal, Sundeep

Harmatz, Paul R.

Harris, William Thomas

Harvey, Jillian

Harville, Emily

Hasan, Shabih U.

Haschke, Manuel

Hasegawa, Tomonobu

Haus, Jacob

Hay, Susanne

Hayde, Nicole

Heerman, William J.

Henriksson, Pontus

Henry, Kate

Henshall, David

Henter, Jan-Inge

Herberg, Ulrike

Hermansson, Ann

Hernandez-Diaz, Sonia

Herz, Josephine

Herzlinger, Doris

Heyer, Geoffrey

Heyne, Roy

Hibbs, Anna Maria

Hibbs, AnnaMaria

Hillman, Noah $\mathrm{H}$.

Hines, Melissa

Hirono, Keiichi

Hirst, Jonathan

Ho, Roger C.

Hodgson, Joshua Mark

Hoevels-Guerich, Hedwig

Hoffman, Hal

Hoffman, Suma 
Hojsak, Iva

Holford, Nick

Holmgren, Anton

Honig, Lawrence

Horn, Alan

Horne, Rosemary S. C.

Horsch, Sandra

Hoshina, Takayuki

Hostetter, Margaret

Hough, Judith

Hov, Johannes R

Hsu, Leon

Hu, Peng

Huang, Weimin

Huber, Reto

Hulzebos, Christiaan

Hummler, Helmut

Hung, Chih-Hsing

Hunt, Carl

Hunter, Catherine

Hutten, Jeroen

Ibla, Juan

Ichesco, E.

Ilyas, Mohammad

Indic, Premananda

Indolfi, Giuseppe

Ingulli, Elizabeth

Irwin, Michael

Isern, Joan

Isganaitis, E.

Jackson, Jamie

Jacobsohn, David

Jacobson, Sandra

Jadcherla, Sudarshan R.

Jain, Vandana

Jakobsen, Lotte

Jamal, Mohamed A.

Janus, Magdalena

Jape, Gayatri

Jasarevic, Eldin

Jeffery, Alison

Jellema, Reint

Jeng, Michael

Jensen, Erik A.

Jetton, Jennifer

Jilling, Tamas

Jimenez-Rivera, Carolina

Jobe, Alan

John, K. R.

Johnston, Bradley

Jolley, Caroline

Jone, Pei-Ni

Jose, Pedro

Josefson, Jami L.

Judge, Kara

Juliano, Sharon

Juliusson, Petur B.

Juul, Sandra

Kaditis, Athanasios

Kaempf, Joseph W.

Kaiser, Jeffrey

Kallapur, Suhas G.

Kaltonbach, K.

Kamaleswaran, Rishikesan

Kaminski, Beth

Kandasamy, Yogavijayan

Kanegane, Hirokazu

Kang, Hee Gyung
Kannan, Sujatha

Kanter, David

Kapadia, Vishal

Kaseva, Nina

Kashtalap, V. V.

Kaskel, Frederick

Kato, Motohiro

Katz, Tiffany

Kawai, Tomoko

Keijzer, Richard

Kelishadi, Roya

Keller, Guillermo Alberto

Kelly, Claire

Kemp, James

Kemp, Matthew

Kemper, Alex

Keszler, Martin

Khalil, Markus

Khan, Asaduzzaman

Khandelwal, Priyanka

Khokha, Mustafa

Kinuthia, John

Kirby, Russell

Klebanoff, Mark

Kleber, Christine

Klentrou, Panagiota

Klevebro, Susanna

Knauf, Claude

Kochi, Cristiane

Koehler, Raymond

Koenig, Joyce

Kojima, Seiji

Kolísko, Martin

Koltsova, Ekaterina

Konduri, Girija

Korevaar, Tim I. M.

Korzeniewski, Steven

Kozinetz, Claudia

Krall, Jenna

Kramer, Boris

Kramer, Boris W.

Kraske, Susanne

Kremer, Leontine

Kribs, Angela

Kristensen, Kurt

Krogulska, Aneta

Kuban, Karl

Kukkonen, Kaarina

Kumar, Ashok

Kumar, Seema

Kwok, Charlie H. T.

Kwong, Amanda

Kyng, Kasper

Lacher, Martin

Lai, Chih-Cheng

Lakshmanan, Ashwini

Lakshminrusimha, Satyan

Lal, Charitharth

Lalani, Seema

Lam, C. W.

Lamot, Lovro

Lane, Shelly J.

Langer, Thomas

Lanphear, Bruce

Lara, D. A.

Larijani, Bagher

Larsen, Britta A.

Latal, Beatrice
Laughon, Matthew

Lautz, Andrew

Lawrence, Shelley

Lei, Wei-Te

Lei, Yunping

Leme, Ana Carolina

Leuridan, Elke

Lev, Hadar

Levy, A.

Levy, Philip

Lewandowski, Adam

Lewis, Tamorah

Li, Marilyn

Li, Yanqi

Lichenstein, Richard

Licht, Daniel

Lin, Jing

Lin, Ming-Tai

Linebarger, Jennifer

Lingappan, Krithika

Lintonjua, Augusto

Liu, Cindy

Liu, Hanmin

Liu, Pi

Liu, Yan

Liu, Yang

Liu, Zhongchen

Lo, Camilla

Lo, Louise

Lo, Warren

Lodha, Abhay

Logan, John

Logue, Catherine

Lombès, Marc

Long, Thomas

Lord, James D.

Losty, Paul

Lu, Jing

Lu, Peng

Lucas, Raquel

Lui, Kei

Luo, Zhong-Cheng

luzuriaga, katherine

Mabeta, Peace

MacLean, J. E.

Macleish, Sarah

Macleod, Malcolm Robert

Macy, Michelle

Madan, Juliette

Maguire, Sabine

Maheshwari, Akhil

Maier, Rolf

Maisels, M. Jeffrey

Maitre, Nathalie

Malheiros, Jackeline

Mallamaci, Antonello

Mallard, Carina

Mamone, Gianfranco

Manary, Mark J.

Marchisio, Paola

Mariani, Thomas

Markel, Troy

Marlow, Neil

Maron, Jill

Marschik, Peter

Marshall, Jeanne

Martens, Dries S.

Martin, Richard
Martinelli, Massimo

Martinez-Vizcaíno, Vicente

Mascarello, Keila

Massa, Valentina

Massaro, An

Masumoto, Junya

Mathur, Amit

Matinolli, Hanna-Maria

Matsell, Douglas

Matthews, Lillian

Matute, Juan

May, Philip

Mayerl, Christopher

Mazarati, Andrey

Mazor-Karsenty, Tal

McCallion, Naomi

McDavid, Lolita

McDonald, Courtney

McDonald, Denise M.

McElroy, Steven

McEvoy, Cindy

McGrath-Morrow, Sharon

McGregor, Alistair

McGuire, Bill

McKenzie, Lara

McKnight, Robert

Medina, Alexandre

Medina-Gomez, Carolina

Meinzen-Derr, Jareen

Menezes, KÃania

Menezes-Filho, Jose A.

Menon, Shina

Merhar, Stephanie

Merianos, Ashley L.

Mericq, Veronica

Mersha, Tesfaye

Mertens, Luc

Meyer, Haakon E.

Meyer, Sascha

Mihatsch, Walter A.

Mikhailov, Theresa

Mikrogeorgiou, Alkisti

Milani, Gregorio

Miller, Steven

Miller, Suzanne

Milligan, Gregg

Minamisawa, Susumu

Mir, Imran

Mishra, Om P.

Mitra, Subhabrata

Moavero, Romina

Mofenson, Lynne

Mohamed, Afifah

Moharir, Mahendranath

Molloy, Luke

Moltu, Sissel

Montaldo, Paolo

Monte, Massimo Dal

Moody, Emily

Mooney, Sandra

Moore, James

Moore, Sophie

Moore, Zena E. H.

Moorman, Randall

Morag, Iris

Morais, Simone

Moreno, Luis

Morgan, Colin 
Morrow, Ellen

Morty, Rory

Moss, Timothy

Motawea, Hanaa

Moxey-Mims, Marva

Msall, Michael

$\mathrm{Mu}$, Dezhi

Muensterer, Oliver

Mukerji, A.

Mukhopadhyay, Sagori

Mulkey, Sarah

Muniraman, Hemananda

Munzer, Tiffany

Mupere, Ezekiel

Murgia, Xabier

Murki, Srinivas

Muro, Andres

Murray, Deirdre

Musial, Kinga

Mynard, Jonathan

Nailescu, Corina

Nair, Jayasree

Najaf, Tasnim

Nakagawara, Akira

Nakanishi, Toshio

Nakhmani, Arie

Narayan, lyer

Naulaers, Gunnar

Nawaytou, Hythem

Neil, Jeffrey

Nelson, Karin

Nemeth, Elizabeta

Neshteruk, Cody

Nestrasil, Igor

Neu, Josef

Neubauer, Vera

$\mathrm{Ni}$, Yen-Hsuan

Niatsetskaya, Zoya

Nicholl, Analise

Nielsen, Heber

Niess, Jan Hendrik

Nightingale, Scott

Nimbalkar, Somashekhar

Nishinakamura, Ryuichi

Nogee, Lawrence

Non, Amy

Northington, Frances

Nosarti, Chiara

Notarangelo, Luigi D.

Nourbakhsh, Noureddin

Novak, lona

Novotny, Edward

Nowicki, Michal

Null, Donald

Nuyt, Anne Monique

O' Gorman, Ruth

Oberhoffer, Renate

Oberklaid, Frank

O'Connell, Amy

Oda, Arata

Ohls, Robin

Oken, Emily

Oliva, Salvatore

Ong, Ken

Ono, Noriaki

OReilly, Michael

O'Riordan, Mary Ann

Owen, Louise S.
Paes, Bosco

Pammi, Mohan

Paneth, Nigel

Papadopoulou, alexandra

Papanna, Ramesha

Parazzini, Fabio

Park, Jason Y.

Parker, William

Parrington, Mark

Partridge, Colin

Pasanen, Anu

Patel, Ravi

Patel, Aloka

Patel, Neil

Pathipati, Praneeti

Patole, Sanjay

Paul, David A.

Paula-Ribeiro, Marcelle

Paulsen, Megan

Paulus, Frank

Peebles, Stokes

Peeples, Mark

Pengcheng, Xun

Pensabene, Licia

Perens, Elliot

Perez, Marta

Perng, Wei

Perrone, Serafina

Peterson, Courtney

Petrarca, Laura

Peyvandi, Shabnam

Pfeiffer, Beth

Philips, Joseph

Phillips, Raylene

Pieper, R.

Pierro, Agostino

Pillas, Demetris

Pillekamp, Frank

Pinney, Sara

Pipaon, Miguel

Poets, Christian

Poindexter, Brenda

Polglase, Graeme

Polin, Richard

Popova, Antonia

Poppe, Tanya

Portella, Andre Krumel

Porter, Forbes

Porter, Jr., George

Portman, Michael

Posod, Anna

Pozo, Oscar J.

Premkumar, Muralidhar

Prince, Lawrence

Prince, Jose

Procaccini, Claudio

Procianoy, Renato S.

Prudente, Sabrina

Prunskaite-

Hyyryläinen, Renata

Puelles Rodriguez, Victor

Pugni, Lorenza

Puopolo, Karen M.

Purvis, Gareth

Quach, Jon

Quadros, Teresa Maria

Bianchini de

Querfeld, Uwe
Rada, Balazs

Radu, Madalina

Rahmel, Tim

Raia, Valeria

Ramakrishnaiah, Raghu

Ramos, Carlos

Randis, Tara

Ranger, Manon

Rao, Rakesh

Rao, Shripada

Ravelli, Angelo

Raz, Sarah

Raza, Syed Asif

Razak, Abdul

Reber, Kris

Reese, Jeff

Reidy, Kimberly

Remmers, Elaine

Ren, Aiguo

Rhee, Christopher

Rhee, Kay

Rhoads, J. Marc

Ribes, Antonia

Richardson, Susan

Richter, Gresham

Richter, Jute

Rigby, Michael J.

Riordan, Sean

Roberts, Jesse

Roberts, Megan

Robinson, Daniel

Rochow, Niels

Rodriguez, Ana

Rodriguez, G.

Rogawski, Elizabeth T.

Rogers, Lynette

Rogers, Elizabeth

Rogerson, Stephen

Rogol, Alan

Roll, Claudia

Romano, Claudio

Romano, Megan

Ronkainen, Eveliina

Rooij, Dirk G. de

Rose, Sean

Rosenblum, Norman

Rossini, Paolo Maria

Rozance, Paul

Rubini, Michele

Rudloff, Silvia

Ruiz, Jonatan

Runyan, Desmond

Ryan, Anthony

Rychik, Jack

Sabir, Hemmen

Safder, Osama

Saha, Gautam Kumar

Saito, Hiroshi

Saki, Najmaldin

Sakowicz, Agata

Sakurai, Kenzo

Salas, Ariel

Salnikova, Lyubov

Salomone, Alberto

Salvatore, Silvia

Samaşca, Gabriel

Samimi, Amir

Sampath, Venkatesh
Sanchez-Solis, Manuel

Sandercock, Gavin

Sangild, Per

Sankar, Raman

Sant'Anna, Guilherme

Santos, Fernando

Sarr, Ousseynou

Sasser, Tyler

Sauer, Pieter

Saugstad, Ola

Savarino, Vincenzo

Scala, Melissa

Schaechter, Judy

Schaible, Thomas

Scharf, Rebecca

Scheinost, Dustin

Schelonka, Robert

Schenkel, Konstantin

Schlapbach, Luregn J.

Schlaudecker, Elizabeth

Schmidt, Barbara

Schmitz, Thomas

Schoberer, Mark

Schoch, Jennifer

Schreiber, Michael

Schwaberger, Bernhard

Scott, Chris

Sears, Kim

Sehgal, Arvind

Sellers, Zachary

Senoo, Makoto

Serin, Halil Ibrahim

Shah, Birju

Shah, Prakesh

Shah, Samir

Shaikh, Nader

Shankaran, Seetha

Shapiro, Steven

Sharfstein, Joshua

Shaw, Dennis

Shaw, Robin M.

Shawber, Carrie

Sheikh, Farah

Shekhawat, Prem

Shelton, Elaine

Sherman, Philip

Sheward, Richard

Shoaff, Jessica

Silke, Bernard

Sim, Xueling

Simeoni, Umberto

Simoes-e-Silva, Ana Christina

Simon, Amos

Simpao, Allan F.

Singer, Georg

Singer, Lynn

Singh, Rachana

Singh, Pratibha

Skeath, Thomas

Slaughter, Jonathan

Slaughter, Laurel

Slusher, Tina M.

Smith, Phillip

Smith, Jodi

Smyser, Christopher

Smyth, lan

Snyder, Susan

Sodhi, Chhinder 
Sola-Visner, Martha

Soll, Roger

Soni, Priya

Soriano-Guillen, Leandro

Sorio, Claudio

Sortica da Costa, Cristine

Soul, Janet

Southard-Smith, Michelle

Spagnuolo, Maria Immacolata

Speleman, Frank

Spencer, Nick

Spittle, Alicia J.

Sporea, loan

Spracklen, Cassandra

Srinivasan, Lakshmi

Sriram, Sudhir

Srivastava, R. N.

Staffler, Alex

Staples, Amy

Stapleton, Bruder

Stark, Michael

Starling, A. P.

Stevenson, David

Stewart, Christopher

Stierman, Bryan

Stockwell, Melissa S.

Stonestreet, Barbara

Stoops, Christine

Stormon, Michael

Strasburger, Victor

Strisciuglio, Caterina

Stroustrup, Annemarie

Stucin Gantar, Irena

Sturm, Ekkehard

Su, Yi-Fong

Suazo, Jose

Sullivan, Jackie

Sun, Xingmin

Suresh, Gautham

Sutherland, Scott

Suzuki, Daisuke

Swanson, Jonathan

Swart, Joost Frans

Sweeney, Emma

Sweeney, Tim

Szajewska, Hania

Szamotulska, Katarzyna

Szilagyi, Moira

Tachtsidis, Ilias

Taglauer, Elizabeth

Talati, Ajay

Talavera, Maria

Talge, Nicole

Tan, Can

Tanaka, Yoichi

Tanski, Susanne

Tattarano, Maria Luisa

Tayebi, Nahid

Taylor, Sarah

Tayman, Cuneyt

Teng, Ru-Jeng
Tharner, Anne

Thayyil, Sudhin

Thebaud, Bernard

Thomas, Diana

Thompson, Loren

Thornton, Claire

Thorup, Jorgen

Thyagarajan, Balamurugan

Ting, Joseph Y.

Tiozzo, Caterina

Tipple, Trent

Tiribelli, Claudio

Toet, Mona

Toet, Alexander

Tomkinson, Grant

Toruner, Cagri

Trabelsi, Mediha

Trachtman, Howard

Trittmann, Jennifer

Troncone, Riccardo

Tsai, Michael Y.

Tschiedel, Eva

Tskitishvili, Ekaterine

Tsuchida, Tammy

Tsugawa, Naoko

Tsukahara, Hirokazu

Tulloh, Robert

Tung, Yi-Ching

Turcotte, Lucie

Turner, Anne

Tyson, Jon

Ueno, A.

Uli, Naveen

Ulinski, Tim

Underwood, Mark

Underwood, Mark

Uziel, Yosef

Vajro, Pletro

Valeriani, Massimiliano

Vali, Payam

Van Bel, Frank

van de Looij, Yohan

van den Akker, Chris

van den Anker, Johannes

van den Hoogen, Nynke

Van Elburg, Ruurd

van Goudoever, Johannes

van Rooij, Iris

van Schaik, Ron

van Weissenbruch, Mirjam

Vandenplas, Yvan

Vannucci, Susan

Vasar, Eero

Vazquez-Zapien, Gustavo

Velikova, Tsvetelina

Vento, Maximo

Verduci, Elvira

Verjans, Georges

Vermeij, Winfried

Vesoulis, Zachary

Vetter-Laracy, Susanne
Vezzani, Annamaria

Vianna, Lauro

Vilan, Ana Isabel

Violano, Pina

Viswanathan, Anuradha

Vitale, Serena

Vitek, Libor

Vockley, Gerard

Volker, Mall

Vollmer, Brigitte

Volovelsky, Oded

Vrijens, Karen

Wacker Gußmann, Annette

Waerden, Judith van der

Wagner, Carol

Wainwright, Mark

Wajner, Moacir

Wald, Martin

Wallace, Joseph Michael

Wallingford, Mary

Walsh, Brian

Waltenberger, Johannes

Walter, E.

Walther, Frans

Walther, Frans

Walton, Sarah

Wambach, Jennifer

Wander, P.

Wang, Bingsong

Wang, David

Wang, George

Wang, Jianshe

Wang, Jou-Kou

Wang, Mingbang

Wang, Xiaobin

Wang, Yan

Wanner, Nicola

Ward, Leigh

Warner, Barbara

Waseem, Muhammad

Watterberg, Kristi

Wear, Keith A.

Weaver, Connie

Weiss, Scott

Weitkamp, Joern-Hendrik

Weitkamp, Joern-Hendrik

Welak, Scott

Welch, Martha G.

Wennberg, Richard

White, Robert

White-Traut, Rosemary

Widhalm, Kurt

Wiliiams, Scott

Willems, Mark Elisabeth Theo-

dorus

Williams, Bethany

Wilschanski, Michael

Wilson, Karen

Wilson-Costello, Deanne

Winter, Harland

Wisgrill, Lukas
Wisnowski, Jessica L.

Witteman, Jurriaan

Wlodarczyk, bogdan

Wlodek, Mary

Wolf, Cordula

Wolf, Martin

Wolfe, A.

Wolff, Mary

Wong, Ronald

Woolf, Alan

Wright, Charlotte

Wright, Clyde

Wright, Robert

Wright, Rosalind

Wu, Shu

$\mathrm{Wu}$, Jia Feng

Wu, June

Wu, Tai-Wei

Wu, Yvonne

Wynn, James

Xia, Liang

Xie, Linglin

Yalcindag, Ali

Yanbakan, Serpil

Yang, Zeng-Jin

Yano, Ikuko

Yap, Hui-Kim

Yawno, Tamara

Yeung, Edwina

Yi, Ming

Young, Karen

Young, Leslie

Younge, Noelle

Yusuf, Kamran

Zaldivar, Frank

Zani, Augusto

Zappitelli, Michael

Zarean, Maryam

Zemlin, Michael

Zempleni, Janos

Zeng, Lin

Zevit, Noam

Zhang, Jun

Zhang, Ke-xiong

Zhang, Lemeng

Zhang, Mingyu

Zhao, Wei

Zheng, Shan

Zheng, Yufang

Zhou, Chao

Zhou, Jichun

Zhu, Changlian

Ziaee, Vahid

Zijlstra, Andries

Zilbauer, Matthias

Zimmerman, Jerry

Zoldan, Janeta

Zurita, Esther 